\title{
Associations of Pericardial Fat Area Determined by Routine Chest Computed Tomography With Coronary Risk Factors and Coronary Artery Disease
}

\author{
Reo Yoshikawa ${ }^{a}$,, Hidekatsu Yanaia, b, c, Sumie Moriyama ${ }^{a}$, Narihiro Furugaki ${ }^{b}$
}

\begin{abstract}
Background: Pericardial fat has been reported to be associated with coronary risk factors and severity of coronary arterial lesions. Previous studies had measured pericardial fat volume (PFV) by using contrastenhanced cardiac computed tomography (CT) when pericardial fat was quantified. We determined pericardial fat area (PFA) in the cross section with the height of sternal angle by using routine chest CT.
\end{abstract}

Methods: We picked up 252 patients who underwent chest and abdominal CT, and we selected patients whose coronary arteries were evaluated by coronary angiography or coronary CT. Coronary artery disease (CAD) was defined as more than $75 \%$ lumen stenosis. PFA was defined as any pixel with CT attenuation of -150 to -30 Hounsfield Unit (HU) within the pericardial sac at the sternal angle level.

Results: Fifty-three patients were eligible. PFA was significantly larger in men than in women. Serum high-density lipoprotein (HDL)cholesterol level was significantly and negatively correlated with PFA. Hemoglobin A1c and carotid arterial intima-media thickness tended to be positively correlated with PFA. PFA was significantly and nearly $50 \%$ larger in patients with CAD than in patients without CAD. The cut-off value of PFA was $10.4 \mathrm{~cm}^{2}$, and sensitivity and specificity of PFA for CAD were $53.8 \%$ and $88.0 \%$, respectively.

Conclusions: Present study is the first to show a significant association of PFA with gender and CAD. PFA can be determined by routine chest CT, and is simpler and more reproducible, and PFA is more available in a greater number of medical institutes as compared with PFV. Present study also showed a discriminatory value of PFA for CAD comparable to PFV.

Keywords: Coronary artery disease; Coronary risk factor; Gender difference; Pericardial fat area

Manuscript submitted February 14, 2019, accepted March 2, 2019

aDepartment of Internal Medicine, National Center for Global Health and Medicine Kohnodai Hospital, Chiba, Japan

${ }^{b}$ Furugaki Diabetes Clinic, Chiba, Japan

${ }^{\mathrm{c}}$ Corresponding Author: Hidekatsu Yanai, Department of Internal Medicine, National Center for Global Health and Medicine Kohnodai Hospital, 1-7-1 Kohnodai, Ichikawa, Chiba 272-8516, Japan.

Email: dyanai@hospk.ncgm.go.jp

doi: https://doi.org/10.14740/jocmr3782

\section{Introduction}

Recently, pericardial fat can be accurately and reproducibly quantified by contrast-enhanced cardiac computed tomography (CT) [1]. Pericardial fat volume (PFV) has been reported to be associated with coronary risk factors such as blood glucose, lipid levels and blood pressure [2,3]. Pericardial fat secretes adipokines and proinflammatory cytokines such as tumor necrosis factor-alpha and interleukin-6 [4], which has been implicated with the pathogenesis of coronary artery disease (CAD) through possible paracrine interaction with coronary arteries. PFV has been reported to be associated with coronary calcium burden and high-risk coronary lesions such as $>50 \%$ luminal narrowing, positive remodeling and low-density plaque $[5,6]$.

Previous studies had measured PFV by using contrastenhanced cardiac CT when pericardial fat was quantified [1]. Briefly, for PFV measurement, superior boundary was defined as the center of the right pulmonary artery as displayed in the sagittal plane, inferior boundary as diaphragm, with special interest to exclude non-pericardial adipose tissue, anterior boundary as pericardial sac as viewed in the axial plane, and posterior boundary as pericardial sac as viewed in the axial plane. PFV was determined via tracing of the pericardial sac as viewed in the axial image with $10-\mathrm{mm}$ slice thickness.

We determined pericardial fat area (PFA) in the cross section with the height of the sternal angle by routine chest CT but not contrast-enhanced cardiac CT. Although our way of quantification of pericardial fat may be evidently simpler and more reproducible, and more available in a greater number of institutes as compared with the previous way, PFV, the association of PFA with coronary risk factors and predicting ability for CAD were unknown. Here, we studied the association of PFA with clinical variables, coronary risk factors, $\mathrm{CAD}$, cardiac systolic and diastolic function and atherosclerosis among Japanese people.

\section{Materials and Methods}

\section{Data source and participants}

Recruitment of patients studied was shown in Figure 1. We picked up 252 patients who underwent routine chest and abdominal CT at Furugaki Diabetes Clinic from April 2017 to 


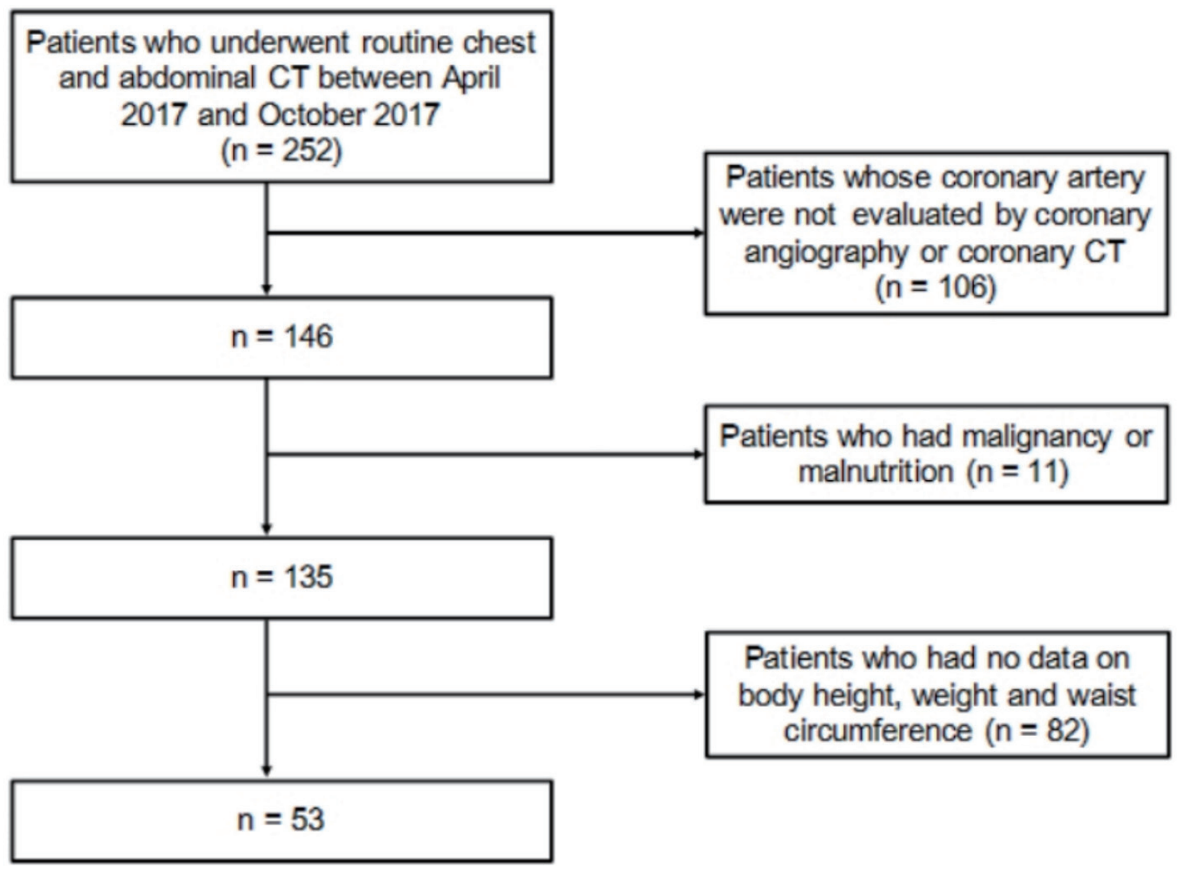

Figure 1. Recruitment of patients studied.

October 2018, and we selected patients whose coronary arteries were evaluated by coronary angiography (CAG) or coronary CT within 3 years. We excluded patients who had suffered from malignancy or malnutrition, and patients who had no data on body height, weight and waist circumference. Diabetes was defined as taking insulin therapy or oral anti-diabetic drugs, or showing a fasting glucose $\geq 126 \mathrm{mg} / \mathrm{dL}$ or non-fasting glucose $\geq 200 \mathrm{mg} / \mathrm{dL}$. Hypertension was defined as showing systolic blood pressure $\geq 140 \mathrm{~mm} \mathrm{Hg}$, or diastolic blood pressure $\geq 90$ $\mathrm{mm} \mathrm{Hg}$, or taking anti-hypertensive drugs. Dyslipidemia was defined as showing low-density lipoprotein-cholesterol (LDLC) $\geq 140 \mathrm{mg} / \mathrm{dL}$, high-density lipoprotein-cholesterol (HDL-C) $<40 \mathrm{mg} / \mathrm{dL}$, triglyceride $\geq 150 \mathrm{mg} / \mathrm{dL}$, or taking anti-lipidemic drugs. CAD was defined as more than $75 \%$ lumen stenosis in coronary arteries evaluated by CAG or coronary CT.

\section{CT scan and pericardial, visceral and subcutaneous fat quantification}

Study subjects underwent routine chest and abdominal CT on a multi-slice CT scanner (Alexion ${ }^{\mathrm{TM}}$, Canon Medical Systems Company, Japan). Tube voltage was $120 \mathrm{kVp}$ with multi-slice scanning. The slice thickness was $5 \mathrm{~mm}$ for multi-slice CT. Pericardial fat quantification was calculated using the software which CT had built in. PFA was defined as any pixel with CT attenuation of -150 to -30 Hounsfield Unit (HU) within the pericardial sac at the sternal angle level (National Center for Global Health and Medicine (NCGM) method). The reasons for choosing the cross section at the height of the sternal angle as the measurement of PFA included that this section is easy to identify by bifurcation of trachea and is not susceptible to the influence of the heart lying down by sub-diaphragmatic fat
(Fig. 2). Visceral and subcutaneous fat areas were determined at the navel level of abdominal CT.

\section{Cardiac function evaluated by chest X-ray and echocardi-} ography

Cardiomegaly was evaluated by using cardio-thoracic ratio on chest X-ray. Cardiac systolic and diastolic functions were

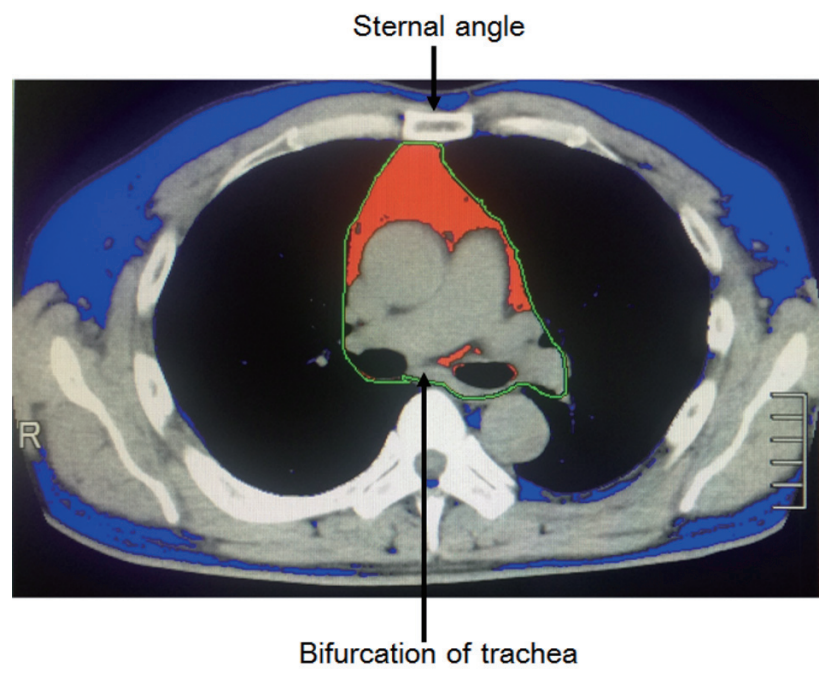

Figure 2. Evaluation of PFA by routine chest CT (NCGM method). PFA was defined as any pixel with CT attenuation of -150 to $-30 \mathrm{HU}$ within the pericardial sac at the sternal angle level. Red and blue areas indicate pericardial fat and other fat areas, respectively. PFA: pericardial fat area. 
evaluated by ejection fraction, E/A (the ratio of E wave (early diastolic filling velocity) to A wave (atrial filling velocity)) and E/e' (the ratio of E wave (early diastolic filling velocity) to e' wave (peak early diastolic velocity)), respectively, measured by echocardiography.

\section{Atherosclerosis evaluated by carotid ultrasonography}

We measured the intima-media thickness (IMT) of internal carotid artery, as the marker for atherosclerosis by using carotid ultrasonography.

\section{Statistical analysis}

Statistical analyses were performed by using JMP, version 9.0.29 (SAS Institute, Inc., Cary, NC, USA). P $<0.05$ and $<$ 0.1 were regarded as statistically significant, and having tendency, respectively. Differences in PFA were determined by a Student's $t$-test. Correlation was analyzed by Spearman rank correlation. The ability of PFA to accurately diagnose CAD was evaluated by a receiver operating characteristic (ROC) analysis. The optimal cut-off values of PFA were calculated by plotting the true-positive rate (sensitivity) against the false-positive rate (1-specificity) when the maximum accuracy (sensitivity plus specificity) was achieved, also called the Youden index.

\section{Ethics statement}

The study protocol was approved by the Medical Ethics Committee of the National Center for Global Health and Medicine (Reference No. NCGM-G003118-00), and this study was performed in accordance with the Declaration of Helsinki.

\section{Results}

\section{Characteristics of study patients}

Fifty-three patients were eligible. Characteristics of study patients were shown in Table 1 . Almost $60 \%$ of patients were male, and mean age was 75 years old. Approximately half of patients had CAD, and $75 \%, 47 \%$ and $81 \%$ of patients had diabetes, hypertension and dyslipidemia, respectively.

\section{PFA, women versus men}

PFA was significantly larger in men $\left(9.75 \mathrm{~cm}^{2}\right)$ than in women $\left(5.43 \mathrm{~cm}^{2}\right)$ (Fig. 3).

\section{PFA among patients with diabetes, hypertension and dyslipidemia}

Comparisons in PFA between patients with and without dia- betes, hypertension and dyslipidemia were shown in Figure 4. There were no significant differences in PFA between patients with and without such diseases.

\section{PFA in patients with and without CAD}

PFA was significantly larger in patients with CAD $\left(9.48 \mathrm{~cm}^{2}\right)$ than in patients without CAD $\left(6.26 \mathrm{~cm}^{2}\right)$ (Fig. 5). The ROC analysis of PFA for CAD was shown in Figure 6. The cut-off value of PFA was $10.4 \mathrm{~cm}^{2}$. Sensitivity and specificity of PFA for $\mathrm{CAD}$ were $53.8 \%$ and $88.0 \%$, respectively. Area under the curve (AUC) was 0.697 (95\% confidence interval (CI): 0.434 $-0.822)$.

\section{Correlation of PFA with metabolic parameters, cardiac function and atherosclerosis}

Body mass index (BMI), waist circumference, subcutaneous and visceral fat area were significantly and positively correlated with PFA (Table 2). Serum HDL-C level was significantly and negatively correlated with PFA. Hemoglobin A1c level and IMT tended to be positively correlated with PFA. However, PFA was not significantly correlated with markers for cardiac systolic and diastolic function. Multiple regression coefficients of PFA with parameters were shown in Table 3. Only waist circumference was significantly correlated with PFA.

\section{Discussion}

In previous studies on pericardial fat, the quantification of PFV has been used. For the measurement of PFV, reconstructed CT data sets were used. PFV was measured using a threshold based method, and an attenuation of -190 to -30 HU was corresponding to adipose tissue in contrast-enhanced cardiac CT scans [1]. PFV was defined as any pixel within these attenuation thresholds inside the pericardial sac. A region of interest was manually drawn following the pericardial sac, starting at the mid-level of the right pulmonary artery.

Jang et al quantified PFA by using low-dose lung CT and they chose the middle part of heart to measure PFA, and they showed significant associations of PFA with fasting blood glucose, triglyceride, HDL-C and blood pressure [7] in healthy adults under 60 years of age. They analyzed ROC curve of PFA for waist circumference, and the AUC was 0.79 (95\% CI: $0.73-0.84)$. Kim et al quantified PFA by using low-dose lung $\mathrm{CT}$ and they chose the mid-ventricular level to measure PFA, and they showed significant association of PFA with the metabolic syndrome in asymptomatic men $(n=130$; mean age, 50.2 \pm 10.77 years) [8], and they analyzed ROC curve of PFA for metabolic syndrome, and the AUC was 0.808 (95\% CI: 0.702 - 0.914). The association between PFA and metabolic coronary risk factors has been reported; however, there were no studies which reported the association of PFA with gender and CAD. Further, previous studies chose the middle part of heart 
Table 1. Characteristics of Patients Studied $(n=53)$

\begin{tabular}{|c|c|c|}
\hline Sex, male & $\mathrm{n}(\%)$ & $31(58.5 \%)$ \\
\hline Age (years) & mean $\pm \mathrm{SD}$ & $75.2 \pm 7.5$ \\
\hline Body height (cm) & mean $\pm \mathrm{SD}$ & $158.0 \pm 11.0$ \\
\hline Body mass index $\left(\mathrm{kg} / \mathrm{m}^{2}\right)$ & mean $\pm \mathrm{SD}$ & $23.9 \pm 3.9$ \\
\hline Waist circumference $(\mathrm{cm})$ & mean $\pm \mathrm{SD}$ & $88.1 \pm 11.6$ \\
\hline Hypertension & $\mathrm{n}(\%)$ & $25(47.1 \%)$ \\
\hline Dyslipidemia & $\mathrm{n}(\%)$ & $43(81.1 \%)$ \\
\hline Coronary artery disease & $\mathrm{n}(\%)$ & $28(52.8 \%)$ \\
\hline \multicolumn{3}{|l|}{ Fat area measured by CT } \\
\hline \multicolumn{3}{|l|}{ Lipids and glucose metabolism } \\
\hline Serum triglyceride $(\mathrm{mg} / \mathrm{dL})$ & mean $\pm \mathrm{SD}$ & $116.6 \pm 73.0$ \\
\hline Serum LDL-C (mg/dL) & mean $\pm \mathrm{SD}$ & $96.2 \pm 20.2$ \\
\hline Serum HDL-C (mg/dL) & mean $\pm \mathrm{SD}$ & $55.5 \pm 18.5$ \\
\hline Hemoglobin Alc (\%) & mean $\pm \mathrm{SD}$ & $6.8 \pm 1.1$ \\
\hline Finding measured by chest X-ray, cardio-thoracic ratio (\%) & mean $\pm \mathrm{SD}$ & $50.4 \pm 6.7$ \\
\hline \multicolumn{3}{|l|}{ Findings measured by echocrdiography } \\
\hline Ejection fraction $(\%)$ & mean $\pm \mathrm{SD}$ & $62.3 \pm 9.4$ \\
\hline $\mathrm{E} / \mathrm{A}$ & mean $\pm \mathrm{SD}$ & $0.87 \pm 0.30$ \\
\hline
\end{tabular}

CT: computed tomography; E/A: the ratio of $E$ wave (early diastolic filling velocity) to A wave (atrial filling velocity); E/e': the ratio of $E$ wave (early diastolic filling velocity) to e' wave (peak early diastolic velocity); HDL-C: high-density lipoprotein-cholesterol; LDL-C: low-density lipoprotein-cholesterol.

to measure PFA.

We determined PFA in the cross section with the height of the sternal angle by using routine chest CT for the first time (NCGM method), because this section is easy to identify by bifurcation of trachea and is not susceptible to the influence of the heart lying down by sub-diaphragmatic fat. Although our way of quantification of pericardial fat is considered to be evidently simpler and more reproducible as compared with the previous way [1], the association of PFA with coronary risk factors and predicting ability for CAD was largely unknown.

Our study showed that men had significantly larger PFA than women. Mancio et al also reported that men had higher PFV than women $(137 \pm 65.6$ vs. $106 \pm 65.6 \mathrm{~mL}, \mathrm{P}<0.01)$ [9], showing that our result by PFA was consistent with the previous result by PFV. Body composition differs between men and women, and men are more likely to accumulate adipose tissue around the trunk and abdomen, whereas women usually accumulate adipose tissue around the hips and thighs [10]. In male patients with CAD, site-related differences of adipose tissue in expression of androgen receptor and aromatase which is capa- ble of converting androgens to estrogens were observed [11]. The association between aromatase expression in pericardial fat and cardiovascular diseases has been suggested [12]. Such different aromatase expression may produce gender difference in pericardial fat.

In our study, BMI was significantly and positively correlated with PFA, showing an agreement with previous studies by PFV, which were performed in Asian and American populations $[2,13]$. Both subcutaneous and visceral fat areas were significantly and positively correlated with PFA; however, the correlation of PFA with visceral fat area was stronger than that with subcutaneous fat area. Waist circumference was the most crucial determinant of PFA in Japanese populations. In the comparison in PFA between patients with and without diabetes, hypertension and dyslipidemia, there were no significant differences in PFA between patients with and without such diseases. However, serum HDL-C level was significantly and negatively correlated with PFA, and hemoglobin A1c tended to be positively correlated with PFA. Such associations between PFA and metabolic parameters also agreed with associations 


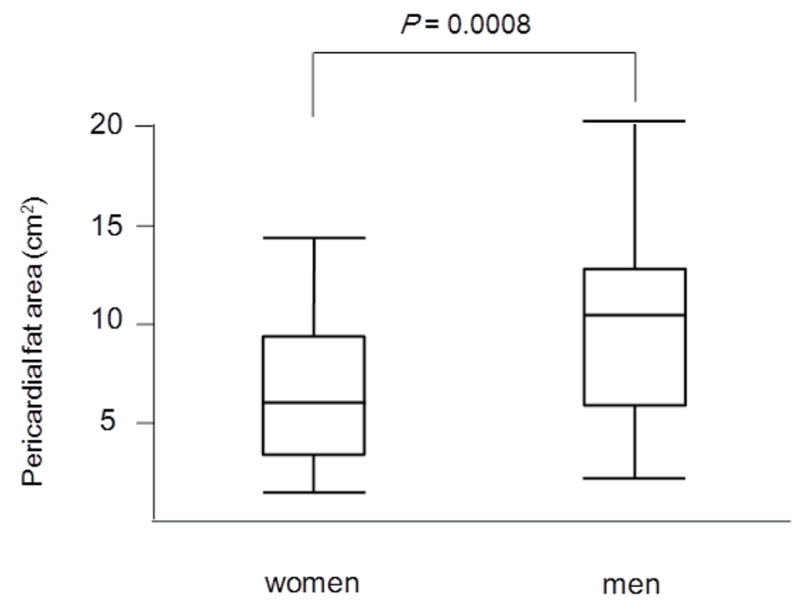

Figure 3. Median levels of PFA between women and men. PFA was significantly larger in men $\left(9.75 \mathrm{~cm}^{2}\right)$ than in women $\left(5.43 \mathrm{~cm}^{2}\right)$. PFA: pericardial fat area.

between PFV and metabolic parameters observed in previous studies $[2,13]$.

PFV was reported to be independently associated with impaired myocardial systolic function and worse left ventricular diastolic function $[14,15]$. Therefore, we studied the as-

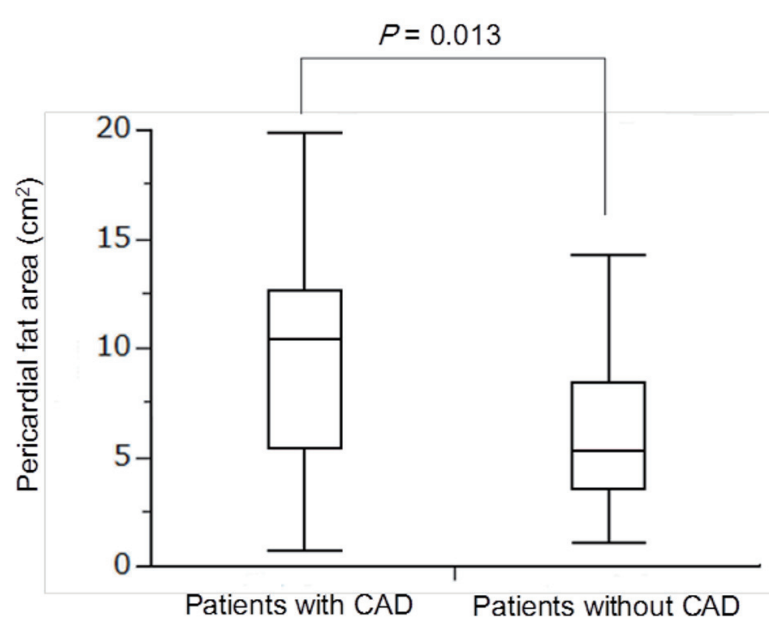

Figure 5. Median levels of PFA between patients with and without CAD. PFA was significantly larger in patients with CAD $\left(9.48 \mathrm{~cm}^{2}\right)$ than in patients without CAD $\left(6.26 \mathrm{~cm}^{2}\right)$. PFA: pericardial fat area; CAD: coronary artery disease.

sociation of PFA with cardiomegaly, systolic function (ejection fraction measured by echocardiography), and diastolic function (E/A and E/e'). However, we could not find any significant correlations of PFA with cardiomegaly, cardiac systolic

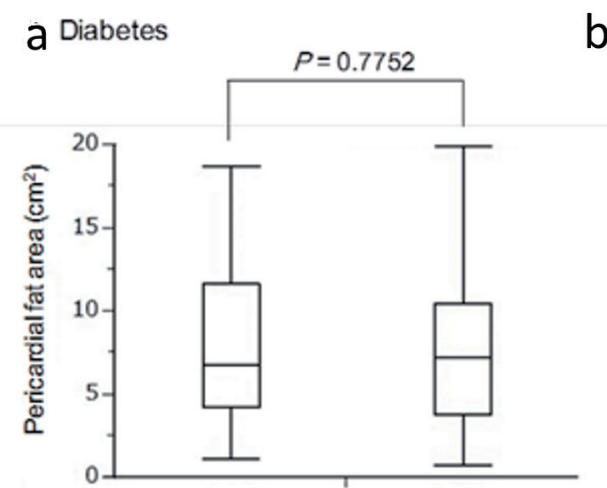

$(+)$ b Hypertension

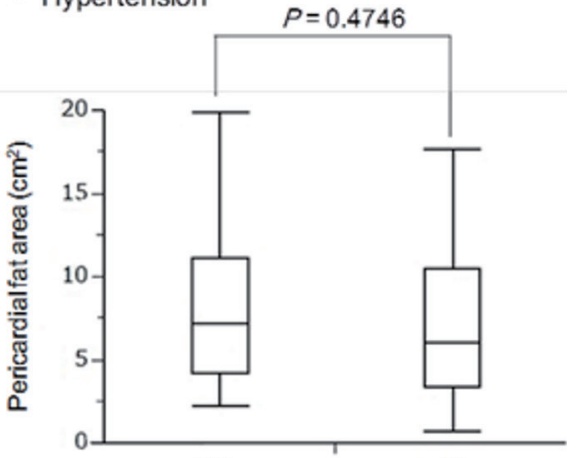

$(+)$
$(-)$

\section{C}

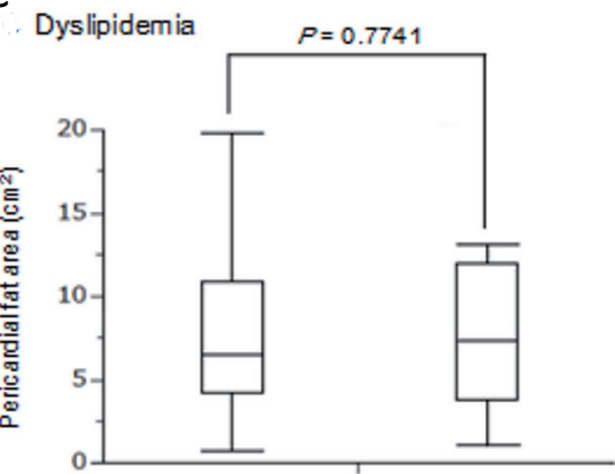

$(+)$

$(-)$

Figure 4. Median levels of pericardial fat area between patients with (+) and without (-) diabetes (a), hypertension (b) and dyslipidemia (c). 


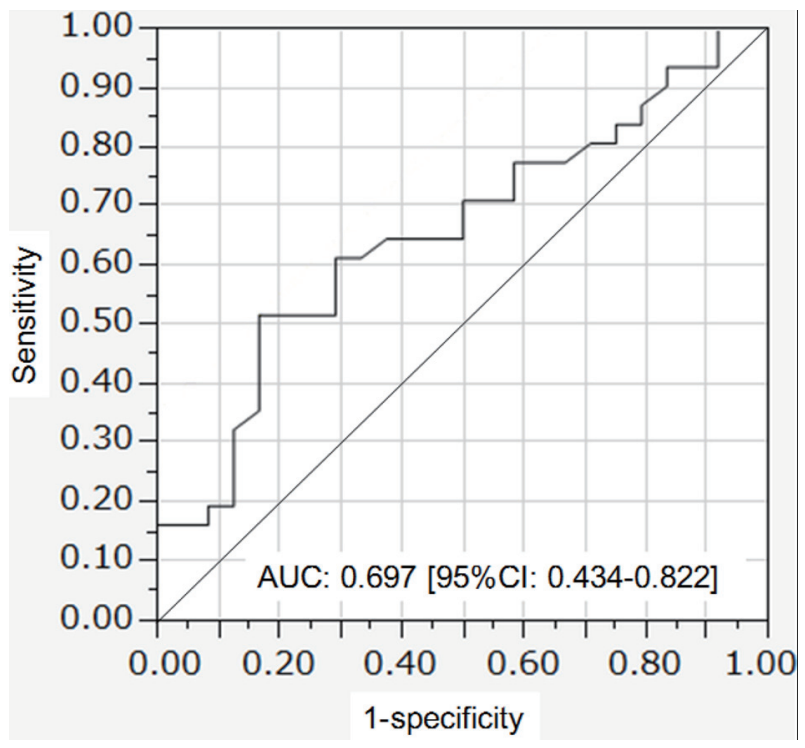

Figure 6. ROC curve of pericardial fat area to predict coronary artery disease. ROC: receiver operating characteristic.

and diastolic function. We cannot explain whether this discrepancy between PFA and PFV was due to the different participant backgrounds or the different modality to quantify pericardial fat.

The evidence on the association between pericardial fat and IMT is very limited. We found only one study by Soliman et al [16]. They reported a significant positive correlation between PFV and internal carotid artery-IMT $(r=0.17, \mathrm{P}<$ $0.0001)$. In our study, PFA tended to be positively correlated with IMT ( $\mathrm{r}=0.3, \mathrm{P}=0.0895)$, which was supported the result by Soliman et al and suggested an association between pericardial fat and atherosclerosis progression.

Schlett et al reported that the median PFV was nearly 50\% higher in patients with CAD as compared with patients without CAD [6]. Our result also demonstrated that the median PFA was nearly $50 \%$ higher in patients with CAD as compared with patients without $\mathrm{CAD}$, showing a complete agreement with the result by Schlett et al. They defined the lesion showing at least two of the following characteristics: spotty calcification, positive remodeling, and low-attenuation plaque as the high-risk lesion [6]. PFV was well discriminative for high-risk lesions (AUC: 0.756 ), and the sensitivity for high-risk lesions was $62 \%$ and the specificity was $84 \%$ [6]. In another study to determine the threshold for the upper limit of PFV to predict major adverse cardiovascular events, ROC curve was constructed by the addition of PFV to Framingham risk score and coronary calcium score [17]. The AUC of such constructed ROC curve was 0.714 [17]. Picard et al studied the association between $\mathrm{CAD}$ and epicardial adipose tissue thickness (EAT) which is epicardial fat maximal thickness on left ventricular lateral free wall at the base of the ventricles [18]. By ROC curve analysis, an EAT value $\geq 2.8 \mathrm{~mm}$ best predicted the presence of $>$ $50 \%$ diameter coronary artery stenosis, with a sensitivity and specificity of $46.1 \%$ and $66.5 \%$ respectively (AUC: 0.58 ) [18]. We defined more than $75 \%$ lumen stenosis in coronary arteries confirmed by $\mathrm{CAG}$ or coronary $\mathrm{CT}$ as $\mathrm{CAD}$. The AUC of
Table 2. Correlation Between Pericardial Fat Area and Parameters

\begin{tabular}{lll}
\hline & Correlation coefficient & P value \\
\hline Body mass index & 0.605 & $<0.0001$ \\
Waist circumference & 0.753 & $<0.0001$ \\
Subcutaneous fat area & 0.541 & $<0.0001$ \\
Visceral fat area & 0.74 & $<0.0001$ \\
Serum triglyceride & 0.204 & 0.1423 \\
Serum LDL-C & 0.106 & 0.4519 \\
Serum HDL-C & -0.453 & 0.0007 \\
Hemoglobin A1c & 0.262 & 0.0584 \\
Cardio-thoracic ratio & 0.133 & 0.454 \\
Ejection fraction & -0.214 & 0.1973 \\
E/A & -0.209 & 0.2293 \\
E/e' & 0.196 & 0.6742 \\
Intima-media thickness & 0.3 & 0.0895 \\
\hline
\end{tabular}

E/A: the ratio of $E$ wave (early diastolic filling velocity) to A wave (atrial filling velocity); E/e': the ratio of $E$ wave (early diastolic filling velocity) to e' wave (peak early diastolic velocity); HDL-C: high-density lipoproteincholesterol; LDL-C: low-density lipoprotein-cholesterol.

ROC of PFA to predict CAD was 0.697 , and the sensitivity for CAD was $53.8 \%$ and the specificity was $88.0 \%$, showing that a discriminatory value of PFA for CAD is comparable to PFV and superior to EAT $[6,17,18]$.

We have to mention the limitation of present study. This is a cross-sectional study and only associations but no causality can be derived from the results. The sample size of our patients is limited and validation by a larger number of study subjects is advisable. Given the growing body of evidence suggesting that PFV may be an important measurement, it appears that standardization of PFA measurements is warranted.

\section{Conclusions}

To our knowledge, present study is the first to show a significant association of PFA determined by routine chest CT with gender and CAD. PFA measurement is simpler and more reproducible and more available in a greater number of medical institutes as compared with PFV. Our study also provided a significant discriminatory value of PFA $\left(10.4 \mathrm{~cm}^{2}\right)$ for CAD comparable to PFV.

\section{Acknowledgments}

None to declare.

\section{Financial Disclosure}

Authors have no financial disclosure to report. 
Table 3. Multiple Regression Coefficients of Pericardial Fat Area With Parameters

\begin{tabular}{llll}
\hline & Odds ratio & $95 \%$ confidence interval & P value \\
\hline Age & 1.04 & $0.917-1.186$ & 0.5416 \\
Sex, male & 1.909 & $0.211-22.18$ & 0.564 \\
Body mass index & 1.002 & $1.606-1.626$ & 0.9943 \\
Waist circumference & 1.222 & $1.005-1.580$ & 0.044 \\
Diabetes & 0.445 & $0.025-4.435$ & 0.5052 \\
Hypertension & 1.557 & $0.237-12.39$ & 0.6462 \\
Dyslipidemia & 0.327 & $0.016-3.427$ & 0.367 \\
\hline
\end{tabular}

\section{Conflict of Interest}

The authors declare that they have no conflict of interest concerning this article.

\section{Informed Consent}

Not applicable.

\section{Author Contributions}

RY and HY designed the research. RY, SM, and NF collected data. RY and HY analyzed data, and HY wrote the paper. All authors read and approved the final paper.

\section{References}

1. Nichols JH, Samy B, Nasir K, Fox CS, Schulze PC, Bamberg F, Hoffmann U. Volumetric measurement of pericardial adipose tissue from contrast-enhanced coronary computed tomography angiography: a reproducibility study. J Cardiovasc Comput Tomogr. 2008;2(5):288-295.

2. Rosito GA, Massaro JM, Hoffmann U, Ruberg FL, Mahabadi AA, Vasan RS, O'Donnell CJ, et al. Pericardial fat, visceral abdominal fat, cardiovascular disease risk factors, and vascular calcification in a communitybased sample: the Framingham Heart Study. Circulation. 2008;117(5):605-613.

3. Wang CP, Hsu HL, Hung WC, Yu TH, Chen YH, Chiu $\mathrm{CA}, \mathrm{Lu} \mathrm{LF}$, et al. Increased epicardial adipose tissue (EAT) volume in type 2 diabetes mellitus and association with metabolic syndrome and severity of coronary atherosclerosis. Clin Endocrinol (Oxf). 2009;70(6):876-882.

4. Mazurek T, Zhang L, Zalewski A, Mannion JD, Diehl JT, Arafat H, Sarov-Blat L, et al. Human epicardial adipose tissue is a source of inflammatory mediators. Circulation. 2003;108(20):2460-2466.

5. Yerramasu A, Dey D, Venuraju S, Anand DV, Atwal S, Corder R, Berman DS, et al. Increased volume of epicardial fat is an independent risk factor for accelerated progression of sub-clinical coronary atherosclerosis. Ath- erosclerosis. 2012;220(1):223-230.

6. Schlett CL, Ferencik M, Kriegel MF, Bamberg F, Ghoshhajra BB, Joshi SB, Nagurney JT, et al. Association of pericardial fat and coronary high-risk lesions as determined by cardiac CT. Atherosclerosis. 2012;222(1):129134.

7. Jang HC, Lee HK, Lee H, Cha JG, Kim YS, Cho JH. Analyzing correlation between epicardial fat area and metabolic syndrome risk factor by using low-dose Lung CT. Pak J Med Sci. 2015;31(5):1207-1212.

8. Kim HJ, Lee H, Lee B, Lee JW, Shin KE, Suh J, Park $\mathrm{HW}$, et al. Diagnostic value of using epicardial fat measurement on screening low-dose chest CT for the prediction of metabolic syndrome: A cross-validation study. Medicine (Baltimore). 2019;98(7):e14601.

9. Mancio J, Pinheiro M, Ferreira W, Carvalho M, Barros A, Ferreira N, Vouga L, et al. Gender differences in the association of epicardial adipose tissue and coronary artery calcification: EPICHEART study: EAT and coronary calcification by gender. Int J Cardiol. 2017;249:419-425.

10. Bredella MA. Sex Differences in Body Composition. Adv Exp Med Biol. 2017;1043:9-27.

11. Kalicinska E, Wojtas K, Majda J, Zacharski M, Skiba J, Sliwowski J, Banasiak W, et al. Expression of sex steroid receptors and aromatase in adipose tissue in different body regions in men with coronary artery disease with and without ischemic systolic heart failure. Aging Male. 2018:1-13.

12. Bernasochi GB, Boon WC, Curl CL, Varma U, Pepe S, Tare M, Parry LJ, et al. Pericardial adipose and aromatase: A new translational target for aging, obesity and arrhythmogenesis? J Mol Cell Cardiol. 2017;111:96-101.

13. Kim DS, Ok EJ, Choi BH, Joo NS. The cutoff pericardial adipose tissue volume associated with metabolic syndrome. Korean J Fam Med. 2018;39(5):284-289.

14. Ng AC, Goo SY, Roche N, van der Geest RJ, Wang WY. Epicardial Adipose Tissue Volume and Left Ventricular Myocardial Function Using 3-Dimensional Speckle Tracking Echocardiography. Can J Cardiol. 2016;32(12):1485-1492.

15. Fontes-Carvalho R, Fontes-Oliveira M, Sampaio F, Mancio J, Bettencourt N, Teixeira M, Rocha Goncalves F, et al. Influence of epicardial and visceral fat on left ventricular diastolic and systolic functions in patients after myocardial infarction. Am J Cardiol. 2014;114(11):1663- 
1669.

16. Soliman EZ, Ding J, Hsu FC, Carr JJ, Polak JF, Goff DC, Jr. Association between carotid intima-media thickness and pericardial fat in the Multi-Ethnic Study of Atherosclerosis (MESA). J Stroke Cerebrovasc Dis. 2010;19(1):58-65.

17. Shmilovich H, Dey D, Cheng VY, Rajani R, Nakazato R, Otaki Y, Nakanishi R, et al. Threshold for the upper nor- mal limit of indexed epicardial fat volume: derivation in a healthy population and validation in an outcome-based study. Am J Cardiol. 2011;108(11):1680-1685.

18. Picard FA, Gueret P, Laissy JP, Champagne S, Leclercq F, Carrie D, Juliard JM, et al. Epicardial adipose tissue thickness correlates with the presence and severity of angiographic coronary artery disease in stable patients with chest pain. PLoS One. 2014;9(10):e110005. 